\title{
4-Faktor-Prothrombinkomplex oder Fresh Frozen Plasma zur Antagonisierung von VKA?
}

Fragestellung: Bei Patienten, die mit Vitamin-K-Antagonisten antikoaguliert sind und eine dringende Operation oder einen invasiven Eingriff benötigen, ist bisher nicht geklärt, ob die Gabe eines 4-Faktor-Prothrombinkomplexes (4F-PCC) der Gabe von Fresh Frozen Plasma (FFP) überlegen ist.

Hintergrund: Für Vitamin-K-Antagonisten steht kein spezifisches Antidot zur Verfügung. Die Gabe von Vitamin K ist zwar in der Lage, die INR (International Normalized Ratio) zu normalisieren, doch hierfür werden mehrere Tage benötigt. Im Fall von Blutungen oder notfallmäßig durchgeführten Operationen oder invasiven Eingriffen wird im Moment die Gabe von 3- oder 4F-PCC oder FFP empfohlen. Größere Studien, welche die beiden Therapieansätze miteinander vergleichen, existieren allerdings bisher nicht.

Patienten und Methodik: Es handelt sich um eine multizentrische offene Phase-III-Studie, in die Patienten im Alter über 18 Jahren eingeschlossen wurden, die mit Vitamin-K-Antagonisten behandelt wurden. Einschlusskriterium war die Notwendigkeit eines sofortigen operativen Eingriffs oder einer invasiven Prozedur. Die Patienten erhielten entweder 4F-PCC oder Plasma, wobei die jeweilige Dosierung von der INR und dem Körpergewicht abhingen. Der primäre Endpunkt war eine effektive
Goldstein JN, Refaai MA, Milling TJ Jr et al. Four-factor prothrombin complex con-centrate versus plasma for rapid vitamin Kantagonist reversal in patients needing urgent surgical or invasive interventions: a phase 3b, openlabel, non-inferiority, randomised trial. Lancet 2015; 385: 2077-87
Hämostase und der ko-primäre Endpunkt war eine Reduktion der INR auf unter 1,3 30 Minuten nach dem Ende der Infusion.

Ergebnisse: In die vorliegende Studie wurden 181 Patienten eingeschlossen, von denen 90 4F-PCC und 91 mit

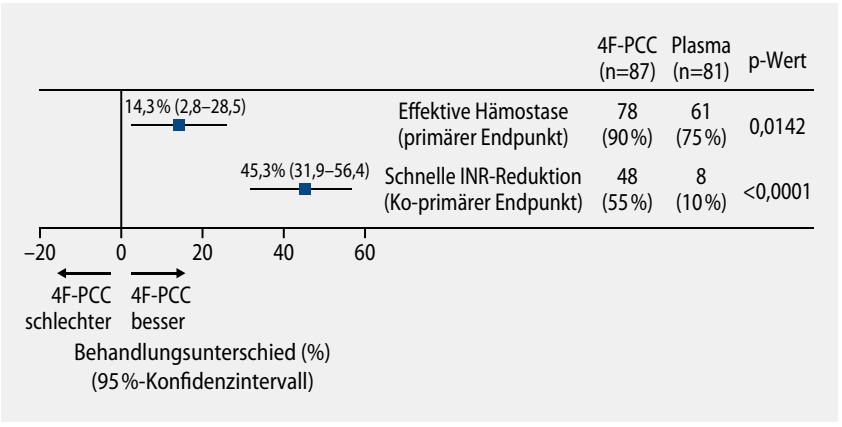

1 Primärer und ko-primärer Endpunkt

FFP behandelt wurden. Die Patienten waren im Mittel 67 Jahre alt. Alle Studienteilnehmer erhielten Vitamin-K-Antagonisten. Die häufigste Indikation für die orale Antikoagulation waren Vorhofflimmern, vaskuläre Erkrankungen und künstliche Herzklappen.

Eine effektive Blutstillung wurde bei $90 \%$ in der 4F-PCCGruppe und bei $75 \%$ in der FFP-Gruppe erzielt ( $\vee$ Abb. 1). Der Unterschied von $14,3 \%$ war statistisch signifikant. Eine Normalisierung der INR wurde bei $55 \%$ in der 4F-PCC-Gruppe, aber nur bei $10 \%$ der Patienten in der FFP-Gruppe erreicht. Auch dieser Unterschied war statistisch signifikant. In der FFP-Gruppe kam es bei $13 \%$ der Patienten zu Symptomen einer Volumenbelastung.

Schlussfolgerungen: Bei antikoagulierten Patienten, bei denen notfallmäßig ein operativer Eingriff oder eine Intervention vorgenommen werden muss, ist zur Normalisierung der Gerinnung die Gabe von 4F-PCC signifikant wirksamer als die Gabe von FFP.

\section{- Kommentar von Hans-Christoph Diener, Essen \\ Prothrombinkomplex ist wirksamer}

Diese außerordentlich wichtige Studie belegt, was bereits in einer Reihe von Leitlinien Eingang gefunden hat, dass nämlich bei Patienten, die mit Vitamin-K-Antagonisten antikoaguliert sind, bevorzugt Prothrombinkomplex (4F-PCC) und nicht FFP verwendet werden sollte. 4F-PCC ist offenbar wirksamer, kann schneller appliziert werden und führt im Gegensatz zu Fresh Frozen Plasma auch signifikant seltener zu einer Volumenbelastung. Dies ist insbesondere bei älteren Patienten mit einer Herzinsuffizienz von großer Bedeutung. Weiterhin gibt es allerdings kein spezifisches Antidot für Patienten, die mit Vitamin-K-Antagonisten antikoaguliert sind.
Konventionelles oder direktes orales Antikoagulans?

Eine orale Antikoagulation kann heute mit Vitamin-K-abhängigen oder neuen, nicht Vitamin-K-abhängigen oralen Antikoagulanzien (NOAK) durchgeführt werden. Es ist daher möglich, Patienten in den Entscheidungsprozess zur Wahl des Antikoagulans einzubinden (5486894).

Diesen Artikel finden Sie, indem Sie den Titel oder die in Klammern gesetzte ID-Nummer in die Suche eingeben. 